\title{
Black hole stereotyping: Induced gravito-static polarization
}

\author{
Barak Kol \\ Racah Institute of Physics, Hebrew University, \\ Jerusalem 91904, Israel \\ barak_kol@phys.huji.ac.il \\ Michael Smolkin \\ Perimeter Institute for Theoretical Physics, \\ Waterloo, Ontario N2L 2Y5, Canada \\ msmolkin@perimeterinstitute.ca
}

\begin{abstract}
We discuss the black hole effective action and define its static subsector. We determine the induced gravito-static polarization constants (electric Love numbers) of static black holes (Schwarzschild) in an arbitrary dimension, namely the induced mass multipole as a result of an external gravitational field. We demonstrate that in $4 \mathrm{~d}$ these constants vanish thereby settling a disagreement in the literature. Yet in higher dimensions these constants are non-vanishing, thereby disproving (at least in $d>4$ ) speculations that black holes have no effective couplings beyond the point particle action. In particular, when $l /(d-3)$ is half integral these constants demonstrate a (classical) renormalization flow consistent with the divergences of the effective field theory. In some other cases the constants are negative indicating a novel non-spherical instability. The theory of hypergeometric functions plays a central role.
\end{abstract}




\section{Contents}

1 Introduction 1

2 The black hole effective action $\quad 4$

$\begin{array}{lll}3 & \text { Measuring Love } & 7\end{array}$

4 Microscopic computation $\quad 8$

$\begin{array}{lll}4.1 & \text { Gravitational polarization } & 11\end{array}$

$\begin{array}{lll}4.2 & \text { Discussion } & 12\end{array}$

$\begin{array}{llr}5 & \text { Diverging Love } & 14\end{array}$

$\begin{array}{llr}6 & \text { EFT side } & 16\end{array}$

$\begin{array}{lll}7 & \text { Summary of results } & 20\end{array}$

$\begin{array}{ll}\text { A Matching normalization constant } & 22\end{array}$

B Useful formulae $\quad 23$

\section{Introduction}

The word "stereotype" carries a negative connotation for judging an individual based on a group to which he or she belongs rather than basing on actual individual traits. ${ }^{1}$ Yet, in this work we shall describe a useful treatment of compact objects including black holes which can be considered as stereotyping (see figure 1).

Most of this paper is devoted to a determination of the coefficients of induced gravitostatic polarization, known as the electric Love numbers [2], ${ }^{2}$ of a black hole in $4 \mathrm{~d}$ and in higher dimensions. Consider a non-trivial gravito-static space-time and consider placing a small non-spinning black hole at an equilibrium point. As a result of the tidal gravitational force the black hole will deform, mass multipole moments will be measurable from far away, and could be interpreted as changes in the internal mass distribution of the black hole. To leading order the induced multipole moment is proportional to the external field multipole at the equilibrium point and we shall refer to the constant of proportionality as the coefficient of induced gravito-static polarization, or Love number.

\footnotetext{
${ }^{1}$ Stereotype (dictionary definition [1]) - A preconceived and oversimplified idea of the characteristics which typify a person, situation, etc.

${ }^{2}$ Named after the British mathematician A. E. H. Love who defined them first in 1911 in the context of Newtonian gravity.
} 


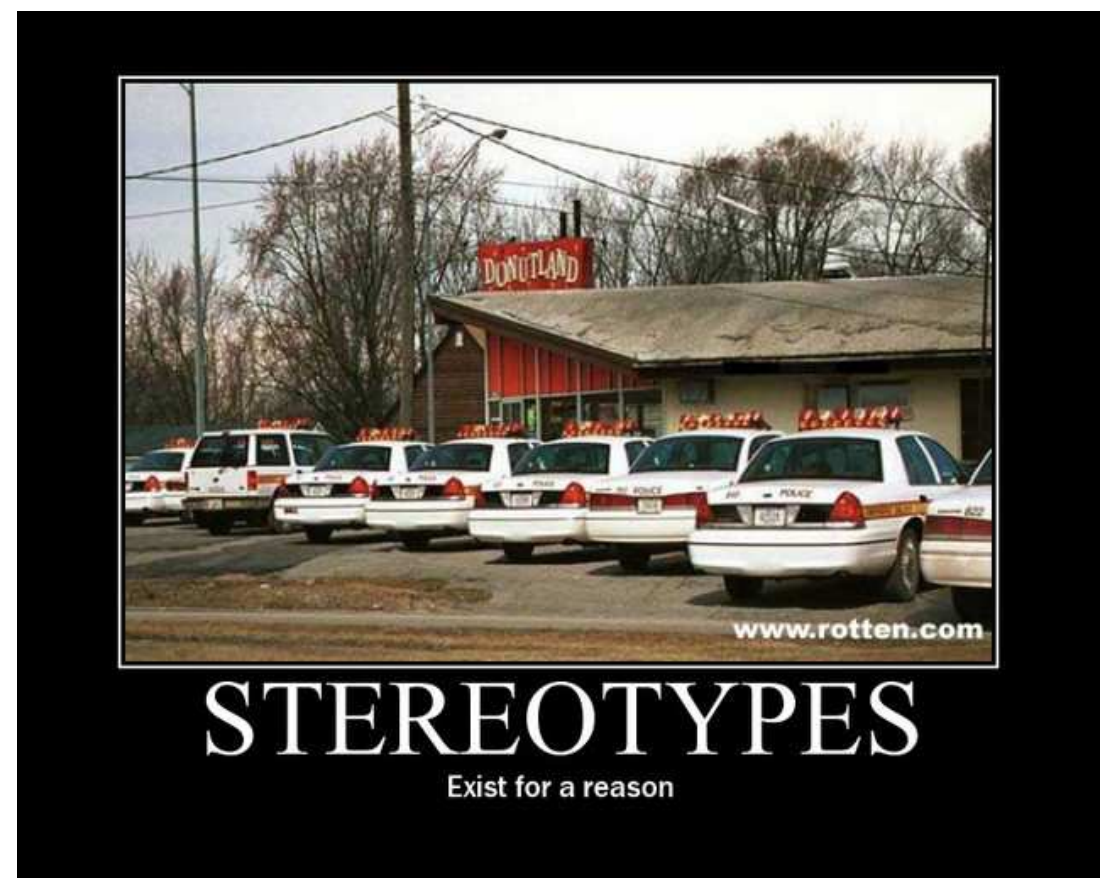

Figure 1.

As a concrete example, consider two very massive and approximately static bodies (see figure 2) . On the line joining them there is a point of gravito-static equilibrium. We place a small $\mathrm{BH}$ at that point and wish to measure the induced mass multipole moments from far away. Another concrete example, one which is exactly static, is a caged black hole, namely a small black hole within a larger extra dimension (see for example [3],[4],[5] and reference therein). In this case the black hole reacts to a gravitational field created by itself (through its "mirror images").

These constants are basic linear response coefficients for black hole physics, much like compressibility in elasticity. Apart for the intrinsic interest, knowing these constants would have several applications. First, they are needed for solving the binary motion. While for $4 \mathrm{~d}$ black holes such effects appear first (in the two body effective action) only at order 5PN, they are much more relevant for other types of compact objects and moreover in very high dimension the leading effect for BHs appears already at order $1^{+} \mathrm{PN}$, namely only a little over 1PN. Another possible application is the study of extended black objects in higher dimensions and their instabilities, as we shall find out.

Even though black hole Love numbers could have been defined following Schwarzschild's discovery of the black hole metric [6] it was only rather recently that works on the subject appeared. [7] computed the second Love number for various models of neutron stars and discussed the possibility of measuring it using gravitational wave detectors. Then in June 2009 three works appeared $[8,9,10]$ which addressed the black hole Love numbers, yet two issues remained open

- While both $[8,9]$ independently found that a certain calculation yields zero contribu- 


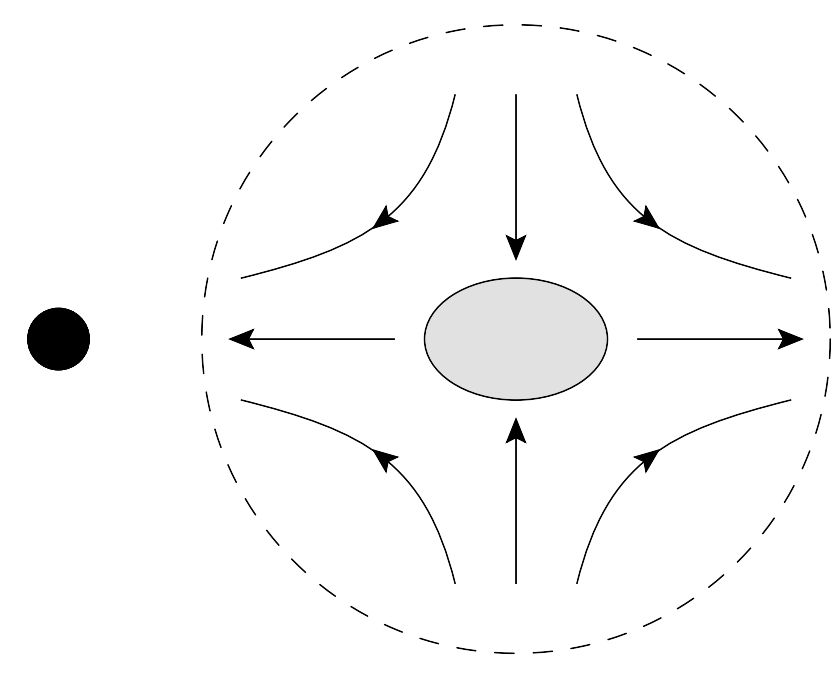

Figure 2. Experimental set-up for measuring the induced gravito-static polarization constants. The black hole (gray) is positioned at an equilibrium point between two fixed stars (black). Its mass multipole moments are measured on a far away envelope (dashed sphere).

tion to the Love numbers, they disagreed on whether another contribution should be included, one arising from the effective field theory side and which was too difficult to compute at the time. ${ }^{3}$

- [8] made an interesting speculation that a black hole may have no non-minimal worldline couplings whatsoever. ${ }^{4}$

We shall consider the determination of the Love numbers from the point of view of the effective field theory approach to GR $[11,12]$ including the ideas of $[4] .{ }^{5}$ We started working on this topic over two years ago but were held back by the above-mentioned apparent ambiguity in their definition.

The strategy of this paper is to calculate the Love numbers for an arbitrary space-time dimension following the idea of "dimension as a parameter of GR", see [14] and references therein including [15]. We shall discover an interesting dependence which will also shed new light on the $4 \mathrm{~d}$ issues.

We start in section 2 by discussing the concept of the black hole effective action and defining its static subsector. We define the induced polarization constants. In section 3 we describe a concrete physical set-up for its measurement together with the correspond-

\footnotetext{
${ }^{3}$ [8] wrote "The question of computing the 'correct' value of $k_{l}$ [the Love numbers] for a black hole is a technically much harder issue which involves investigating in detail the many divergent diagrams that enter the computation of interacting point masses at the 5-loop (or 5PN) level." while [9] "boldly proclaimed" that the tidal Love numbers of a black hole must be zero. Finally [10] wrote "there are subtleties inherent in any definition of the multipole moments of $\mathrm{BHs}$, so that there is currently no unambiguous determination of the $k_{l}$ Love number of BHs."

4 "This vanishing suggests, but in no way proves, that the effective action describing the gravitational interactions of black holes may not need to be augmented by nonminimal worldline couplings." [8]

${ }^{5}$ See [13] for early precursors of the EFT approach to GR.
} 


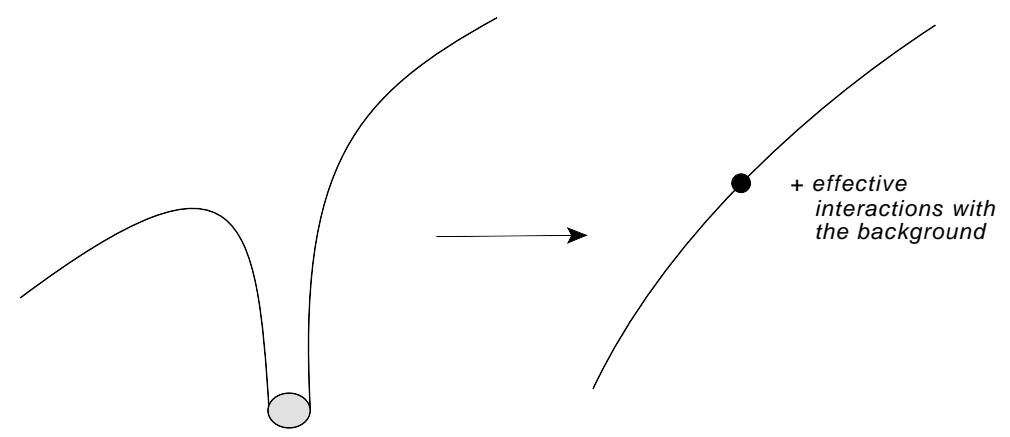

Figure 3. The idea behind the black hole effective action: the full black hole space-time geometry is replaced by a point particle together with some effective interactions with its slowly varying background.

ing computation. In section 4 we perform the "microscopic" calculation, namely we find the appropriate non-asymptotically-flat deformation of the Schwarzschild solution and we determine the Love numbers. The results are interesting and we proceed to discuss them in subsection 4.2. In section 5 we discuss divergences and their physical meaning including counter-terms, classical RG flow and cancellation of divergences in the EFT. In section 6 we look at the calculation from an EFT perspective and reproduce several results with Feynman diagrams. We end with a summary and discussion in section 7 .

\section{The black hole effective action}

Consider a soliton of size $r_{0}$ moving in a background with typical length scale $L \gg r_{0}$. Given the hierarchy of scales it is natural to use the point particle approximation. However, this approximation is certainly limited, as it does not retain almost any of the object's physical properties, namely the finite-size effects. To improve upon that one uses either a matched asymptotic expansion between a near zone and a far zone, or an effective field theory (EFT) approach which is the more modern tool (see for example [4] which followed the EFT approach [11]). The idea is illustrated in figure 3.

A black hole $(\mathrm{BH})$ is a soliton of interest in Einstein's theory of gravity. In [11] a matching procedure was outlined which produces the black hole effective action. Later [4] defined it essentially ${ }^{6}$ as follows

$$
\begin{aligned}
S_{\text {full }, e f f}\left[x^{\mu}, e_{A}^{\mu} ; Q_{\text {int }} ; g\right] & =S_{E F T, \text { eff }}\left[x^{\mu}, e_{A}^{\mu} ; Q_{\text {int }} ; g\right] \\
:=S_{\text {bulk }}\left(g_{B H}\left(x^{\mu}, e_{A}^{\mu} ; Q_{\text {int }} ; g\right)\right) & :=S_{\text {bulk }}[g]+S_{B H}\left[x^{\mu}, e_{A}^{\mu} ; Q_{\text {int }} ; g\right]
\end{aligned}
$$

This definition means that the effective theory is built such that its effective action for a point particle in a given background, is identical with the same effective action in the full

\footnotetext{
${ }^{6}[4]$ did not list the internal degrees of freedom as they appear here - those were later discussed in $[16,17]$.
} 
theory with a full black hole metric inserted into the same background. Accordingly, given a space-time metric $g$, one must solve for a black hole metric $g_{B H}$ which describes a black hole (namely a horizon) embedded on an appropriate world-line given by $x^{\mu}(\tau)$ in $g$ and having the prescribed spin and internal degrees of freedom, $e_{A}^{\mu}, Q_{\text {int }}$ respectively. The full bulk action (Einstein-Hilbert + gauge fixing) is evaluated on $g_{B H}$. The resulting expression has a (standard) bulk contribution and a contribution localized on the world-line. The black hole effective action is defined to be the latter.

We now wish to define the static sector of $S_{B H}$. In this sector the background metric is static (namely time independent and time reversal symmetric), the black hole location is fixed and it cannot rotate nor excite its internal degrees of freedom. Therefore in this sector

$$
S_{\text {bulk }}\left(g_{B H}(g)\right)=S_{\text {full,eff }}[g]=S_{E F T, e f f}[g]=S_{b u l k}[g]+S_{B H, s t}[g]
$$

and the BH effective action depends only on the background (but not on any collective or internal coordinates). For self-consistency the location of the black hole must be an equilibrium point of the gravitational background, namely a static geodesic, which without loss of generality we take to be the origin, 0 .

In the static limit it is convenient to use the Non-Relativistic Gravitational (NRG) field decomposition $[4,18]$, namely a temporal KK reduction, which replaces the Einstein field $g_{\mu \nu}$ by three fields: the Newtonian potential $\phi$, the gravito-magnetic vector potential $A_{i}$ and the spatial metric $\gamma_{i j}$ according to

$$
d s^{2}=e^{2 \phi}\left(d t-A_{i} d x^{i}\right)^{2}-e^{-2 \phi / \hat{d}} \gamma_{i j} d x^{i} d x^{j}
$$

where we conveniently denote

$$
\hat{d}:=d-3
$$

In the static limit $A_{i}=0$.

We define a Lagrangian by

$$
S_{B H, s t}[\phi, \gamma]=\int d t \mathcal{L}_{B H, s t}[\phi, \gamma]
$$

We expand $\mathcal{L}_{B H, s t}$ around flat space-time $\gamma_{i j}=\delta_{i j}, \phi=0$. In general the leading term in $S_{B H}$ is the universal point-particle action

$$
\begin{aligned}
S_{B H} & =-m \int d \tau+\ldots \\
d \tau & :=\sqrt{g_{\mu \nu}(x) d x^{\mu} d x^{\nu}}
\end{aligned}
$$

Therefore in the static sector

$$
\mathcal{L}_{B H, s t}^{(0)}=-m
$$

Perturbations around flat space-time are parameterized by $\phi, \sigma_{i j}$ where $\gamma_{i j}=\delta_{i j}+\sigma_{i j}$. Terms proportional to the equations of motion can be removed by a field re-definition. In particular

$$
\partial_{i i} \phi,\left.\partial_{i} \phi\right|_{0}
$$


are redundant terms, the first being a linearized equation of motion in the bulk (assuming a vacuum space-time) and the second being the linearized geodesic equation (no gravitational field at 0 ). We now wish to examine the leading terms beyond the point particle approximation, namely the finite-size corrections. There can be no terms linear in $\phi, \sigma$ due to the spherical symmetry (of the Schwarzschild black hole) - for instance a term linear in $\phi$ would represent a mass multipole for Schwarzschild.

The most general terms quadratic in $\phi$ can be written as follows

$$
\begin{aligned}
\mathcal{L}_{B H, s t} & =-m+\frac{K}{2} \sum_{l=2}^{\infty} \lambda_{l}\left|\partial^{I_{l}} \phi\right|^{2}+\ldots \\
\left|\partial^{I_{l}} \phi\right|^{2} & :=\frac{1}{l !} \sum_{I_{l}} \partial^{I_{l}} \phi \partial^{I_{l}} \phi
\end{aligned}
$$

where $I_{l}:=\left(i_{1}, \ldots, i_{l}\right)$ is a multi-index and each $i$ runs over the spatial directions $i=$ $1, \ldots, d-1$ and $K$ will be defined shortly below in (2.12). We start with $\phi$ (rather than $\sigma_{i j}$ ) because the leading finite-size effect for the post-Newtonian two-body effective action comes from the quadrupole $(l=2)$ term above. ${ }^{7}$ The sums starts with $l=2: l=0$ is absent since $\phi$ is only derivatively coupled, and $l=1$ is absent due to (2.8). We refer to the constants $\lambda_{l}$ as the induced gravito-static polarization constants, or tidal Love numbers. Their normalization here was chosen in a rather natural way, such that (for generic $d, l$ )

$$
Q_{I_{l}}=-\lambda_{l} \phi_{I_{l}} \quad \phi_{I_{l}}:=\left.\partial_{I_{l}} \phi\right|_{0}
$$

where $Q_{I_{l}}$ is the mass multipole at the origin induced by the background field multipole $\phi_{I_{l}}{ }^{8}$ In particular, the factor $\mathrm{K}$ is chosen such that the kinetic term for $\phi$ is $S \supset-K / 2 \int \partial_{i} \phi \partial_{i} \phi$. From(4.8) we have

$$
K:=\frac{1+1 / \hat{d}}{8 \pi G}
$$

The sign convention is such that in weak (Newtonian) gravity $\lambda>0$. Most of this paper is devoted to computing $\lambda_{l}$ for all $d$. The dimensions of $\lambda_{l}$ are

$$
\left[\lambda_{l}\right]=M L^{2 l}=L^{\hat{d}+2 l}
$$

Returning now to our title "black hole stereotyping" we see that according to effective field theory it is useful to "stereotype" a BH when it is viewed from afar by a series of properties each with a specific order. ${ }^{9}$ The mass is most relevant, followed by the spin which is non-static and cannot be seen in (2.9). The next property and the first finite-size one is $\lambda_{2}$, and so on ...

\footnotetext{
${ }^{7}$ In addition in $4 \mathrm{~d} \sigma_{i j}$ cannot fluctuate since in $3 \mathrm{~d}$ space Ricci flatness implies a flat space.

${ }^{8}$ Despite considerable effort so far we were not able to find a clear enough definition of the standard normalization of the tidal Love numbers to allow comparison.

${ }^{9}$ Actually there is some freedom in how we count the order. Apart from derivative counting we may also assign dimensions to the fields $\phi, \sigma$. For instance in $\mathrm{PN} \phi$ should be considered order $m$ while $\sigma$ is order $m^{2}$.
} 


\section{Measuring Love}

Here we translate the general definition of the Love numbers $\lambda(2.9)$ into an experimental measurement set-up and a corresponding computation.

In the experimental set-up, following the description in the introduction, one positions a non-rotating black hole at an equilibrium point of a weak gravito-static field (such that the typical distance for non-linear effects $L_{n l}$ is much larger than $r_{0}$ ). The Newtonian potential $\phi$ is taken to be of the form of a pure $l$-multipole, namely $\phi$ is a homogeneous function of degree $l$ in $x^{i}$ the spatial coordinates and satisfies the Laplace equation $\partial_{i i} \phi=0$. One then measures the induced mass $l$-multipole from the asymptotic form of $\phi,{ }^{10}$ and computes $\lambda$ as the constant of proportionality in (2.11).

The computation starts with a background field $\phi(x)$ satisfying the flat space eq. of motion $\partial_{i i} \phi=0$. As usual we may perform a decomposition into spherical harmonics $\phi=\sum_{l m} \phi_{l m}(r) Y_{l m}(\Omega)$ where in a general dimension $m$ stands for a multi-index. A general solution to the radial equation in flat space is of the form $\phi_{l}=A r^{l}+B r^{-l-\hat{d}}$. Imposing origin regularity we have

$$
\phi_{l}=\bar{\phi} r^{l} .
$$

Now we should obtain $\phi_{B H}$, the field in the presence of a black hole which asymptotes to $\phi$ and is regular on the horizon (for clarity we suppress now the index $l$ ). For $r \ll L_{n l}$ we can write $\phi_{B H}$ as a linear combination

$$
\phi_{B H}(r)=\bar{\phi}\left(\phi_{1}(r)+\hat{\lambda} \phi_{2}(r)\right)
$$

where

$$
\begin{aligned}
& \phi_{1}=r^{l}\left(1+\ldots \mathcal{O}\left(\frac{m}{r^{\hat{d}}}\right)\right) \\
& \phi_{2}=r^{-l-\hat{d}}\left(1+\ldots \mathcal{O}\left(\frac{m}{r^{\hat{d}}}\right)\right)
\end{aligned}
$$

and $\hat{\lambda}$ is some constant.

Considering the equivalent definition of $\lambda(2.11)$, and recognizing that the induced mass multipole at the origin $Q_{I}$ is proportional to $\bar{\phi} \hat{\lambda}$, the coefficient of $\phi_{2}$, while $\phi_{I}$ is proportional to $\bar{\phi}$ we find that

$$
\lambda=N \hat{\lambda}
$$

where the normalization constant is found to be (see appendix A)

$$
N=4 \pi \frac{\pi^{\hat{d} / 2}}{2^{l} \Gamma\left(\frac{\hat{d}}{2}+l\right)} .
$$

We would like to make two comments about our procedure (3.2-3.5).

Possible ambiguity. In the flat space theory $\phi_{1}, \phi_{2}$ in (3.3) have no corrections. However, in the presence of corrections the definition of $\phi_{1}$ becomes ambiguous to adding a multiple

\footnotetext{
${ }^{10}$ Again at distances $r$ such that $r_{0} \ll r \ll L_{n l}$.
} 
of $\phi_{2}$ whenever the powers in the two series (3.3) overlap, namely whenever $(2 l+\hat{d}) / \hat{d} \in$ $\mathbb{N} \equiv 1,2,3, \ldots$ We may express this as

$$
2 \hat{l}+1 \in \mathbb{N}
$$

where we defined

$$
\hat{l}:=\frac{l}{\hat{d}} \equiv \frac{l}{d-3}
$$

Though in general $\hat{l}$ need not be integral, in $4 \mathrm{~d} \hat{l}$ is nothing but $l$, and this ambiguity is essentially the one noted in [10].

A simple and effective solution is to observe that for generic values of $l, d$ no such issue exists, and then the special values can be treated as a limit. Alternatively, one must use the general definition (2.2) to separate the contributions to $\hat{\lambda}$ originating from two different EFT vertices (2.9): the $m$ vertex and the $\lambda$ vertex.

We note that in EFT language the condition (3.6) means nothing but that $\lambda$ has a dimension which is an integral power of $m$.

Later we shall find that the small parameter for the asymptotic expansion (3.3) is actually $m^{2} / r^{2 \hat{d}}$, at least in the background of a free scalar. Hence the condition for possible ambiguity (3.6) becomes more restrictive, namely

$$
\hat{l}+\frac{1}{2} \in \mathbb{N} .
$$

Consistency with earlier terms of the EFT. The general definition of the static effective BH action (2.2) requires that the EFT action reproduces the effective action of the full theory. In the present context it means that corrections to $\phi_{1}$ (at least those which dominate $\phi_{2}$ ) should be reproduced in the EFT by the $m$-vertex alone. Indeed the equation of motion involves the black hole metric which can be fully reproduced from the $m$-vertex while the $\lambda$-vertex reproduces the boundary condition (horizon regularity). In section 6 we shall see several examples for this consistency.

\section{Microscopic computation}

In our first computation we consider a minimally coupled scalar field $\psi$, rather than the Newtonian potential $\phi$. We do that for several reasons (i) this case is simpler (ii) the gravitational case will turn out to be similar and (iii) this case actually describes the master field for tensor mode perturbations [19], namely perturbations of the spatial metric in $d>4$.

The action is

$$
S[\psi]=\frac{1}{2} \int|\partial \psi|^{2}:=\frac{1}{2} \int \sqrt{-g} d^{d} x g^{\mu \nu}(x) \partial_{\mu} \psi \partial_{\nu} \psi
$$

We note that $K_{\psi}=1$ instead of (2.12). We wish to solve for $\psi$ in the background of a $d$ dimensional black hole in Schwarzschild coordinates

$$
\begin{aligned}
d s^{2} & =f d t^{2}-f^{-1} d r^{2}-r^{2} d \Omega_{d-2}^{2} \\
f & :=1-\left(\frac{r_{0}}{r}\right)^{\hat{d}}
\end{aligned}
$$


where $r_{0}$ is the Schwarzschild radius.

It is convenient to change coordinates $r \rightarrow X$ where

$$
X:=\frac{1}{r^{\hat{d}}}
$$

namely $r_{0}^{\hat{d}} X=1-f .^{11}$

Decomposing into (normalized) spherical harmonics the action becomes ${ }^{12}$

$$
S=-\frac{\hat{d}}{2} r_{0}^{\hat{d}} \int d X\left[f\left|\partial_{X} \psi_{l}\right|^{2}+\frac{\hat{l}(\hat{l}+1)}{X^{2}}\left|\psi_{l}\right|^{2}\right]
$$

where $\hat{l}$ was defined in (3.7). The equation of motion is

$$
0=\left[\partial_{X} f \partial_{X}-\frac{\hat{l}(\hat{l}+1)}{X^{2}}\right] \psi_{l}
$$

We note that the equation of motion depends on $d, l$ only through the combination $\hat{l}$. Moreover, this static equation is analytic in $X$ while the time dependent equation contains the term $X^{-2 / \hat{d}}=r^{2}$ which is non-analytic for $d>5$. It would be interesting to explain these simplifications.

Solution. The equation of motion (4.5) has three singularities all of which are regular: at the singularity, the horizon and asymptotically. Therefore the solutions can be expressed by the hypergeometric function. From hereon we use units such that $r_{0}=1$. The solution which is regular at the horizon is

$$
\psi(X)=X^{-\hat{l}} F(-\hat{l},-\hat{l}, 1 ; 1-X)=F(\hat{l}+1,-\hat{l}, 1 ;-(1-X) / X)=P_{\hat{l}}\left(\frac{2-X}{X}\right)
$$

The second equality uses the Pfaff identity (B.4) and $P_{\hat{l}}$ are the Legendre polynomials.

In order to read $\hat{\lambda}$ we must expand around $r=\infty$, namely $X=0$. We do that using (B.5) and we use the Gamma function identities (B.7) to simplify the expression, finally arriving at

$$
\hat{\lambda}_{\psi}=\frac{1}{2^{4 \hat{l}+2}} \frac{\Gamma^{2}(\hat{l}+1)}{\Gamma\left(\hat{l}+\frac{1}{2}\right) \Gamma\left(\hat{l}+\frac{3}{2}\right)} \tan \pi \hat{l} r_{0}^{2 l+\hat{d}}
$$

where we restored units using powers of $r_{0}$.

Isotropic coordinates. At first sight dimensional analysis (3.6) allows for divergences when $2 \hat{l}+1=1,2, \ldots$, yet these occur only for $2 \hat{l}+1=2,4, \ldots$ This suggests that the small parameter can be taken to be $m^{2}$ rather than $m$.

\footnotetext{
${ }^{11}$ We record the standard relation between the Schwarzschild radius $r_{0}$ and the mass $m$, namely $r_{0}^{\hat{d}}=$ $16 \pi G m /\left[(\hat{d}+1) \Omega_{\hat{d}+1}\right]$.

${ }^{12}$ Note that we consider the static limit, therefore the integral over time decouples and we suppress it in what follows.
} 
Indeed, this is the case. The static Einstein-Hilbert action, when expressed in terms of NRG fields (2.3), reads [4]

$$
S\left[\phi, \gamma_{i j}\right]=\frac{1}{16 \pi G} \int \sqrt{-\gamma} d^{d} x\left[-\left(1+\frac{1}{\hat{d}}\right) \gamma^{i j}(x) \partial_{i} \phi \partial_{j} \phi+R[\gamma]\right]
$$

and enjoys the following parity symmetry

$$
\phi \rightarrow-\phi \quad \gamma_{i j} \rightarrow \gamma_{i j}
$$

When supplemented by $m \rightarrow-m$ it is a symmetry also of the relevant interaction term $-m \phi$. In gauges which respect this symmetry, such as the isotropic and the harmonic gauges we may conclude that $\gamma_{i j}=\gamma_{i j}\left(m^{2}\right)$ while $\phi(-m)=-\phi(m)$. Moreover, $\psi$ couples only to $\gamma_{i j}$ as can be seen by expressing its action (4.1) in the static case in terms of NRG fields

$$
S[\psi]=\frac{1}{2} \int \sqrt{-\gamma} d^{d} x \gamma^{i j}(x) \partial_{i} \psi \partial_{j} \psi
$$

Therefore $m^{2}$ is indeed the small parameter for $\psi$, thereby justifying the improved dimensional condition (3.8).

The Schwarzschild gauge which we used here (4.2) provides a simple metric but does not respect this symmetry and therefore this parity property was not manifest. It turns out that there is a different representation of the black hole metric which preserves the parity symmetry while providing equations of motion which are quite similar to Schwarzschild coordinates. These are the isotropic coordinates

$$
\begin{aligned}
d s^{2} & =\left(f_{-} / f_{+}\right)^{2} d t^{2}-f_{+}^{4 / \hat{d}}\left(d \rho^{2}+\rho^{2} d \Omega^{2}\right) \\
f_{ \pm} & :=1 \pm\left(\rho_{0} / \rho\right)^{\hat{d}}
\end{aligned}
$$

This metric is written in NRG form and satisfies the parity property (4.9).

The coordinate transformation which takes us from isotropic to Schwarzschild is given by

$$
r^{\hat{d}}=\rho^{\hat{d}}\left(1+\left(\frac{\rho_{0}}{\rho}\right)^{\hat{d}}\right)^{2}
$$

In particular, the location of the horizon in isotropic coordinates is at $\rho_{0}$ which is related to $r_{0}$ through

$$
\rho_{0}^{\hat{d}}=\frac{1}{4} r_{0}^{\hat{d}} .
$$

The static action in isotropic coordinates now becomes

$$
S=-\frac{\hat{d}}{2} \rho_{0}^{\hat{d}} \int d Z f_{+} f_{-}\left[\left|\partial_{Z} \psi_{l}\right|^{2}+\frac{\hat{l}(\hat{l}+1)}{Z^{2}}\left|\psi_{l}\right|^{2}\right],
$$

where

$$
Z=\left(\frac{\rho_{0}}{\rho}\right)^{\hat{d}}
$$


The solution to the equation of motion which is regular at the horizon is given by ${ }^{13}$

$$
\psi(Z)=Z^{-\hat{l}} F\left(1 / 2,-\hat{l}, 1 ; 1-Z^{2}\right) .
$$

In terms of $\rho_{0}$ our result (4.7) simplifies a bit and becomes

$$
\hat{\lambda}_{\psi}=\frac{\Gamma^{2}(\hat{l}+1)}{\Gamma\left(\hat{l}+\frac{1}{2}\right) \Gamma\left(\hat{l}+\frac{3}{2}\right)} \tan \pi \hat{l} \rho_{0}^{\hat{d}(2 \hat{l}+1)} .
$$

Combining with $(3.4,3.5)$ this completes the computation of the induced polarization constants for the case of a scalar field background.

\subsection{Gravitational polarization}

Our starting point is the master equation for gravitational perturbations of scalar type in $d$ dimensions [20] which are the $d$ dimensional generalization of the Zerilli equation [22] and describe perturbations associated with the Newtonian potential. In our notation the equation reads

$$
\left[\frac{d^{2}}{d X^{2}}+\frac{2(\hat{d}-1) X+2}{\hat{d} X(X-1)} \frac{d}{d X}+\frac{(\hat{d}-1) X-(l-1)(l+\hat{d}+1)}{\hat{d}^{2} X^{2}(1-X)}\right] Y(X)=0
$$

where we continue to suppress the index $l$, namely $Y \equiv Y_{l}$. We change variables into

$$
\hat{Y}:=r Y
$$

and the equation reads

$$
\left[f \partial_{X}^{2}-2 \partial_{X}-\frac{\hat{l}(\hat{l}+1)}{X^{2}}\right] \hat{Y}=0 .
$$

This equation can be gotten from the following action

$$
S[\hat{Y}]=-\int d X\left[f^{2}\left|\partial_{X} \hat{Y}\right|^{2}+\frac{f \hat{l}(\hat{l}+1)}{X^{2}}|\hat{Y}|^{2}\right]
$$

As in the case of a minimally coupled scalar the equation is analytic in $X$ and depends on $l, d$ only through $\hat{l}$. We comment that this action encodes very economically the rather longer Zerilli potential, and that its form suggests that $\hat{Y}$ is the time component of a contravariant vector, namely $\hat{Y} \equiv V^{t}$ for some vector $V$.

The solution is

$$
\hat{Y}(X)=X^{-\hat{l}} F(1-\hat{l},-\hat{l}, 2 ; 1-X)=F(\hat{l}+1,-\hat{l}, 2 ;-(1-X) / X)
$$

Expanding around $X=0$ we find

$$
\hat{\lambda}=-\frac{\Gamma(\hat{l}) \Gamma(\hat{l}+2)}{\Gamma\left(\hat{l}+\frac{1}{2}\right) \Gamma\left(\hat{l}+\frac{3}{2}\right)} \tan \pi \hat{l} \rho_{0}^{\hat{d}(2 \hat{l}+1)}=-\left(1+\frac{1}{\hat{l}}\right) \hat{\lambda}_{\psi}
$$




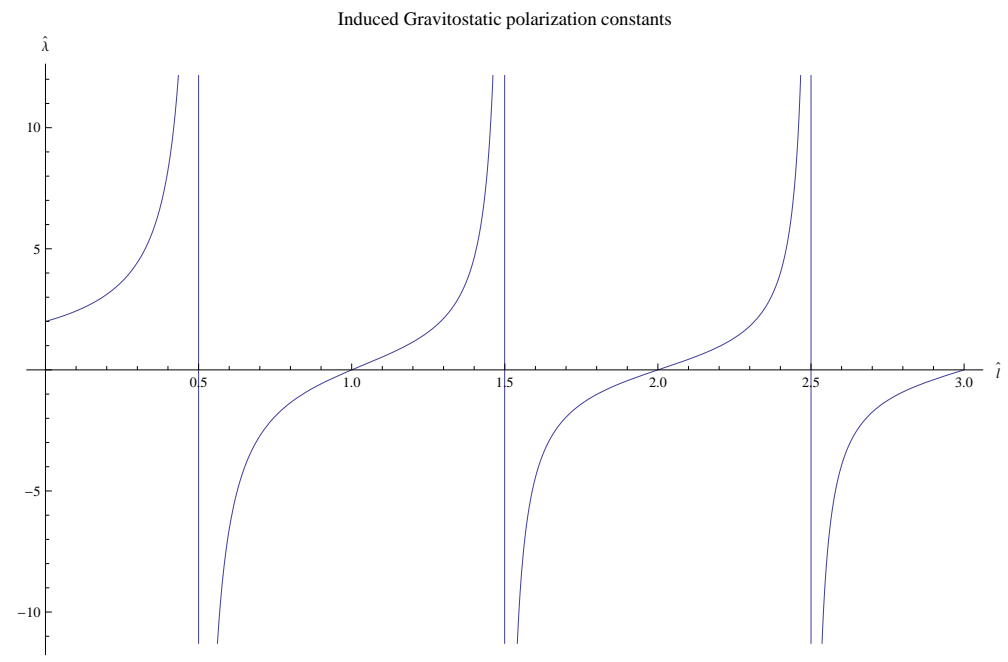

Figure 4. A graph of the raw gravito-static Love numbers $\hat{\lambda}$ of a black hole as a function of $\hat{l} \equiv l /(d-3)$ where $l$ is the spherical harmonic index, and $d$ is the total space-time dimension. Units are such that $\rho_{0}=1$ where $\rho_{0}$ is the location of the horizon in isotropic coordinates (4.13)

where $\rho_{0}$ was defined in (4.13).

The graph of $\hat{\lambda}$ is given in figure 4 .

In order to relate the result for $\hat{\lambda}$ (4.23) with our original definition (2.9) we must find the relation between $\hat{Y}$ and the Newtonian potential $\phi$. When going to the EFT we must take the flat space limit of $\hat{Y}$. Since there is only one gauge invariant scalar the limit of $\hat{Y}$ and $\phi$ must be proportional to each other, namely $\hat{Y} \rightarrow g(r) \phi$ for some $g(r)$. Next we observe that in the flat space limit the equation of motion for $\hat{Y}$ becomes $0=\Delta \hat{Y}$. Comparing with the (flat space) equation of motion of $\phi$, namely $0=\Delta \phi$ we conclude that $g(r)=$ const and actually we may normalize our definition of $\hat{Y}$ such that $g(r)=1$. Altogether we found that in flat space

$$
\hat{Y} \rightarrow \phi
$$

(it would be nice to show this directly from the definition of $\hat{Y}$, see also [23, 24]). Therefore we can proceed and combine (4.23) with the normalization factor $(3.4,3.5)$ to obtain our main result - the induced gravito-static polarization constants or a Schwarzschild black hole.

\subsection{Discussion}

We can now resolve the issues concerning $4 \mathrm{~d}$ Love numbers which were listed in the introduction:

- $4 \mathrm{~d}$ Love numbers (of electric type) vanish.

\footnotetext{
${ }^{13}$ This transformation is known in the mathematics literature as a quadratic transformation of the hypergeometric function, see for example [21].
} 
Indeed in $4 \mathrm{~d} \hat{d}=1, \hat{l}$ is always integral and $\tan \pi \hat{l}=0$. This settles a disagreement in the literature (described in the introduction), validating the intuition of [9]. We note that in general [8] were also right to consider an additional EFT contribution, and indeed we shall find it to be non-zero for half integral $\hat{l}$. Yet in $4 \mathrm{~d}$ it happens to vanish.

Our approach was able to avoid the danger of ambiguity in definition since for generic dimensions none exists, and the $4 \mathrm{~d}$ limit is smooth. Thus it can be considered a dimensional regularization. The $4 \mathrm{~d}$ limit also serves as a first test of our results.

The vanishing of Love numbers is quite surprising especially that the black hole horizon is known to deform, see for example [8, 10, 25, 26]. It implies a certain infinite rigidity of the mass distribution of a $4 \mathrm{~d}$ black at least with respect to linearized gravito-static perturbations.

- Black holes do have non-minimal world-line couplings for $d>4$ (where $\hat{l}$ could be non-integral). This settles negatively the speculation made in the abstract of [8], at least for $d>4$.

The tangent factor in (4.23) has several implications

- $\lambda$ vanishes whenever $\hat{l}$ is integral.

- $\lambda$ has a pole whenever $\hat{l}$ is half-integral. This is related to a classical renormalization flow and will be discussed below. A second test of our result will come from a comparison of the residue of such poles against EFT divergences.

- $\lambda(\hat{l})$ oscillates and changes sign. In particular it can be negative. The interpretation of a negative value is not clear at the moment. A negative compressibility of an elastic material implies an instability. However, a black hole cannot be divided and a negative specific heat, for example, does not imply an instability of a black hole, but rather of the black string [27]. This instability is spherisymmetric but we believe the during pinching this symmetry would be spontaneously broken. We speculate that negative Love numbers are related to this last instability.

- In $5 \mathrm{~d}$ there are only zeros and poles.

We make some additional observations:

- The factor in (4.23) which includes the Gamma functions has quite a smooth behavior for $\hat{l}>0$, for large $\hat{l}$ it approaches 1 and corrections in $1 / \hat{l}$ can be computed

$$
\frac{\Gamma^{2}(\hat{l}+1)}{\Gamma\left(\hat{l}+\frac{1}{2}\right) \Gamma\left(\hat{l}+\frac{3}{2}\right)}=1+\frac{3}{4 \hat{l}}-\frac{3}{32 \hat{l}^{2}}+\mathcal{O}\left(\frac{1}{\hat{l}^{3}}\right) .
$$

This behavior suggests that a theory can be formulated in the $\hat{l} \rightarrow \infty$ limit. 
- At integral values of $\hat{l}$ the solutions are especially simple - they are polynomials in $X$, since when either $a$ or $b$ are $0,-1,-2, \ldots$ the hypergeometric series $F(a, b, c, ; x)$ is finite. A useful explicit formula is

$$
F(c+k, b, c ; X)=\frac{\Gamma(c)}{\Gamma(c+k)} X^{1-c} \frac{d^{k}}{d X^{k}} X^{c+k-1}(1-X)^{-b}
$$

for $k=0,1,2, \ldots$

\section{Diverging Love}

In this section we examine more closely the case where $\hat{l}$ is half integral and both Love numbers $(4.17,4.23)$ diverge. The situation is familiar in quantum field theory where it leads to RG flow, and here it provides another example, quite pleasing, for classical RG flow see $[11,28,29]$.

Cancellation of divergences. While the gravitational $\hat{\lambda}$ diverges (4.23), $\hat{Y}$, the horizon regular wave-function, (4.22) remains finite, of course. Looking at the asymptotic expansion according to (B.5) and substituting $a=1-\hat{l}, b=-\hat{l}, c=2$ for $\hat{Y}$ (4.22) we have

$$
\begin{aligned}
F(1-\hat{l},-\hat{l}, 2 ; 1-X) & =\frac{\Gamma(2) \Gamma(2 \hat{l}+1)}{\Gamma(\hat{l}+1) \Gamma(\hat{l}+2)} F(1-\hat{l},-\hat{l},-2 \hat{l} ; X)+ \\
& +\frac{\Gamma(2) \Gamma(-2 \hat{l}-1)}{\Gamma(1-\hat{l}) \Gamma(-\hat{l})} X^{2 \hat{l}+1} F(\hat{l}+1, \hat{l}+2,2 \hat{l}+2 ; X)
\end{aligned}
$$

The divergence in $\hat{\lambda}$ originates from a divergence in the factor $\Gamma(-2 \hat{l}-1)$ in the second summand. However, at the same time the $c$ parameter in the first hypergeometric function becomes a negative integer $c_{1}=-2 \hat{l} \rightarrow-n$ which implies a divergence in that function. Quite generally the diverging part in such a case is given by

$$
\begin{aligned}
& F(a, b,-n+\epsilon ; X)= \\
= & X^{n+1} \frac{\Gamma(a+n+1) \Gamma(b+n+1)}{\Gamma(a) \Gamma(b) \Gamma(n+2)} \Gamma(-n+\epsilon) F(a+n+1, b+n+1, n+2 ; X)+\mathcal{O}\left(\epsilon^{0}\right)
\end{aligned}
$$

where $n=1,2, \ldots$ and $\Gamma(-n+\epsilon)=(-)^{n} /(n ! \epsilon)+\mathcal{O}\left(\epsilon^{0}\right)$. Using this relation with $a=$ $1-\hat{l}, b=-\hat{l}, n=2 \hat{l}$ one confirms that the two divergences indeed cancel as $\epsilon \rightarrow 0$.

Log terms. As a byproduct of the cancellation of divergences and the presence of dimensional constants a $\log (r)$ term is generated in $\hat{Y}(r)$. Indeed, as the divergences cancel at order $\mathcal{O}\left(\epsilon^{-1}\right)$ the power law $X^{2 \hat{l}+1}=X^{n+1-\epsilon}$ in the second summand (reaction term) generates a finite $\mathcal{O}\left(\epsilon^{0}\right) \log X$ term multiplied by the residue of the pole. Hence the coefficient of the log term, which is interpreted as the beta function, is directly related to the coefficient of divergence (the pole residue in our dimensional regularization).

The same phenomenon is seen also from a solution of the differential equation of motion (4.20) through the substitution of an asymptotic power series $\hat{Y}=\sum_{k} \hat{Y}_{k} X^{k}$. For 
half integral $\hat{l}$ the resulting recursion relation at order $k=2 \hat{l}+1$ becomes $0 \cdot \hat{Y}_{2 \hat{l}+1}=$ source $\neq 0$, which is known from the mathematical theory of differential equations to signal the appearance of log terms.

Finite, RG-flowing part. Next we compute the finite part. To do that, we must separate $\lambda$ into a counter-term and a finite part. The counter-term must cancel the EFT divergence. The finite part depends of course on a choice of renormalization scheme, and we use the standard MS (Minimal Subtraction) scheme. As we saw log terms get generated and accordingly the finite part depends also on the scale in which it is evaluated. So altogether we must keep both the pole residue and the finite part in $\lambda$, but not higher terms in the $\epsilon$ expansion.

For convenience we turn to the case of a free scalar. Let us now assume that $\hat{l}=n / 2$ is half integral, that is $n=1,3,5, \ldots$ Then the induced polarization (4.17) diverges. However, according to (4.16) the wave function $\psi$ is finite. As will be explained later, the divergent part of induced polarization is cancelled by another divergence inherent to the full solution (4.16). This means that $\lambda_{\psi}$ is given by the sum of an infinite part and a finite term which we now turn to compute. To separate these contributions, we introduce the following definitions

$$
m=L^{\epsilon} m_{r}, \quad \epsilon=\hat{d}-\hat{d}_{0},
$$

where $L$ is an arbitrary length scale and $m_{r}$ has length dimension $-\hat{d}_{0}$. Of course, $m$ must be $L$-independent, therefore $m_{r}$ flows with $L$ according to

$$
L \frac{d m}{d L}=0 \quad \Rightarrow \quad L \frac{d m_{r}}{d L}=-\epsilon m_{r}
$$

Substituting these definitions into (4.17) and expanding in $\epsilon$, yields ${ }^{14}$

$$
\begin{aligned}
\lambda_{\psi}= & \frac{N L^{\epsilon} \rho_{0}^{\hat{d}_{0}(n+1)}}{4 \pi} \frac{\Gamma^{2}\left(\frac{n}{2}\right)}{\Gamma\left(\frac{n+1}{2}\right) \Gamma\left(\frac{n+3}{2}\right)} \\
\times & \begin{array}{l}
\times\left(\frac{2 n \hat{d}_{0}}{\epsilon}-2 \frac{n^{2}\left(n \hat{d}_{0}-2\right)-n}{\left(\hat{d}_{0}+1\right)(n+1)}+2 n^{2} H_{\frac{n-1}{2}}-n^{2}\left[2 H_{\frac{n}{2}}+\hat{d}_{0} \log \pi-2 \hat{d}_{0} \log \left(L / \rho_{0}\right)\right]\right. \\
\left.\quad+\hat{d}_{0} n(n+1) \Psi\left(\hat{d}_{0} / 2+1\right)-\frac{\hat{d}_{0} n}{2} \Psi\left(\hat{d}_{0}(n+1) / 2\right)+\mathcal{O}(\epsilon)\right),
\end{array}
\end{aligned}
$$

where $H_{\alpha}$ is the $\alpha$-th harmonic number and $\Psi(z)$ is the digamma function. Notice that we should keep $L^{\epsilon}$ in front of the above expression to maintain correct dimensions for $\lambda_{\psi}$ in $(d+\epsilon)$-dimensional spacetime. In the special case $l=2, \hat{d}_{0}=4(n=1)$, we obtain

$$
\left.\lambda_{\psi}\right|_{l=2, \hat{d}_{0}=4}=\frac{\pi^{3} L^{\epsilon} \rho_{0}^{8}}{3}\left[\frac{1}{\epsilon}+\frac{1}{2}\left(\log \frac{2}{\pi}+\frac{7}{60}-\gamma\right)+\log \frac{L}{\rho_{0}}+\mathcal{O}(\epsilon)\right] .
$$

The pole in $\epsilon$ corresponds to the divergence. In the EFT approach this term represents the so-called counter term which has to be introduced into the theory in order to make the

\footnotetext{
${ }^{14}$ Note that $\rho_{0}$ depends on $\hat{d}$, therefore one has to express it in terms of $m$ first and only then proceed with expansion in $\epsilon$.
} 
observables, e.g. the wave function $\psi$, finite. In contrast, the finite piece in the expression for $\lambda_{\psi}$ represents the renormalized value of $\lambda_{\psi}$, i.e.

$$
\begin{aligned}
& \lambda_{\psi}^{r n}(L)= \frac{N \rho_{0}^{\hat{d}_{0}(n+1)}}{4 \pi} \frac{\Gamma^{2}\left(\frac{n}{2}\right)}{\Gamma\left(\frac{n+1}{2}\right) \Gamma\left(\frac{n+3}{2}\right)} \\
& \times\left(2 n^{2} H_{\frac{n-1}{2}}-2 \frac{n^{2}\left(n \hat{d}_{0}-2\right)-n}{\left(\hat{d}_{0}+1\right)(n+1)}-n^{2}\left[2 H_{\frac{n}{2}}+\hat{d}_{0} \log \pi-2 \hat{d}_{0} \log \left(L / \rho_{0}\right)\right]\right. \\
&\left.\quad+\hat{d}_{0} n(n+1) \Psi\left(\hat{d}_{0} / 2+1\right)-\frac{\hat{d}_{0} n}{2} \Psi\left(\hat{d}_{0}(n+1) / 2\right)\right) .
\end{aligned}
$$

In particular, while $\lambda_{\psi}$ is certainly $L$-independent, the induced polarization $\lambda_{\psi}^{r n}(L)$ exhibits a classical RG flow

$$
\beta_{\lambda_{\psi}}=L \frac{d \lambda_{\psi}^{r n}(L)}{d L}=\frac{2 N \hat{d}_{0}}{\pi} \frac{\Gamma^{2}\left(\frac{n+2}{2}\right)}{\Gamma\left(\frac{n+1}{2}\right) \Gamma\left(\frac{n+3}{2}\right)} \rho_{0}^{\hat{d}_{0}(n+1)} .
$$

\section{$6 \quad$ EFT side}

In this section we demonstrate and confirm the equivalence of the EFT description by mirroring some of the previous matching calculations on the EFT side.

For convenience we consider the case of a free scalar field (4.1) and we choose the isotropic coordinates (4.11).

Integrating out short scales of order $\rho_{0}$, leaves us with the following effective action

$$
S_{E F T, e f f}(\sigma, \phi, \psi)=S_{b u l k}(\sigma, \phi, \psi)+S_{B H, s t}(\sigma, \phi)+\frac{1}{2} \sum_{l=2}^{\infty} \lambda_{l}\left|\partial^{I_{l}} \psi\right|^{2},
$$

where $^{15}$

$$
S_{\text {bulk }}(\sigma, \phi, \psi)=S[\psi]+\frac{\hat{d}(\hat{d}+1)}{64 \pi} \int d x^{\hat{d}+2} \sigma^{\frac{\hat{d}-4}{2}}(\partial \sigma)^{2}-\frac{\hat{d}+1}{16 \pi \hat{d}} \int d x^{\hat{d}+2} \sigma^{\frac{\hat{d}}{2}}(\partial \phi)^{2},
$$

and the indices are contracted with flat metric $\delta_{i j}$. To simplify the gravity action (4.8), we set $\gamma_{i j}=\sigma \delta_{i j}$ for the background metric in isotropic coordinates and used the following transformation rule for the $(d-1)$-dimensional curvature scalar

$$
\gamma_{i j}=e^{2 \omega} \delta_{i j} \Rightarrow R[\gamma]=-e^{-2 \omega}\left[2(\hat{d}+1) \nabla^{2} \omega+\hat{d}(\hat{d}+1) \partial_{i} \omega \partial^{i} \omega\right]
$$

where $\omega=\log (\sigma / 2)$. Note that the full action is quadratic in $\psi$ and symmetric to constant shifts, $\psi \rightarrow \psi+$ const. This explains why only quadratic terms in the derivatives of $\psi$ are present in the above effective action.

In what follows we match the quadrupole $\lambda_{2}$. Introducing the following perturbation

$$
\psi \rightarrow \bar{\psi}+\psi \quad \bar{\psi}=\psi_{i j} x^{i} x^{j}, \quad Q_{i}^{i}=0
$$

\footnotetext{
${ }^{15}$ For simplicity we set $G=1$ for the rest of this section.
} 


$$
\begin{aligned}
& \frac{p}{2}=8 \pi \frac{d-3}{d-2} \frac{1}{p^{2}} \quad \sum_{k_{2}}^{k} m=\frac{d-2}{16 \pi}\left(k_{1} \cdot k_{2}\right) \\
& \underline{p}--\underbrace{k_{1}}_{k_{2}} m=\frac{1}{p^{2}} m=\frac{d-3}{2}\left(k_{1} \cdot k_{2}\right)
\end{aligned}
$$

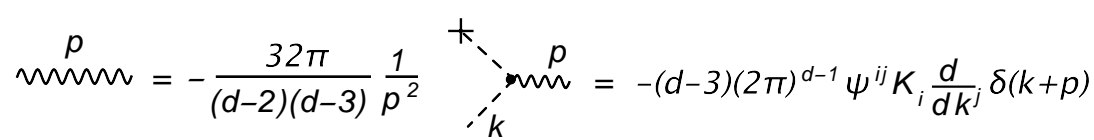

$$
\begin{aligned}
& \|=-m
\end{aligned}
$$

Figure 5. All necessary diagrams to match $\lambda_{2}$ in isotropic coordinates. Solid, dashed and wavy lines represent propagators of $\phi, \psi$ and $\hat{\sigma}$ respectively. Cross indicates insertion of $\bar{\psi}$. Wave numbers flow into the vertex.

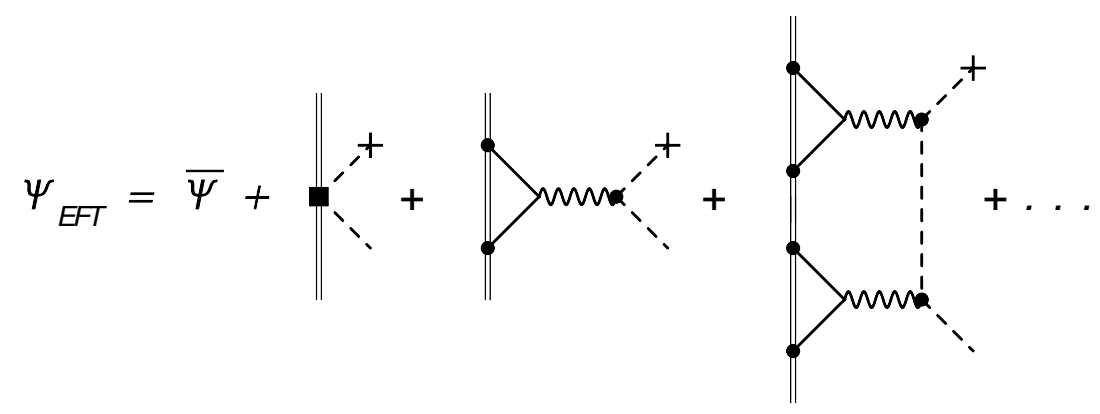

(a)

(b)

(c)

Figure 6. Diagrams contributing to the asymptotic value of the scalar field $\psi$.

into the effective action (6.1), we evaluate the asymptotic expansion of the scalar field, $\psi_{E F T}$, and subsequently match the result with the corresponding expansion of the full solution (4.16). The Feynman rules can be obtained by expanding (6.1) in the weak field approximation, i.e., $\hat{\sigma}=\sigma-1, \phi, \psi \ll 1$. All necessary rules for our needs are listed in figure 5 . In what follows we use dimensional regularization to evaluate various diagrams. All necessary formulas to accomplish the computations can be found, e.g. in the appendices of $[28]$.

The contribution of the induced quadrupole $\lambda_{2}$ to $\psi_{E F T}$ is shown in figure $6(\mathrm{a})$ and is given by

$$
\text { Fig.6(a) }=\frac{\lambda_{2}}{\rho^{\hat{d}+4}} \frac{\Gamma\left(\frac{\hat{d}+4}{2}\right)}{\pi^{\hat{d} / 2+1}} \bar{\psi} .
$$

For $\hat{d} \geq 5$ this contribution dominates all other possible Feynman graphs, since according 
to (2.13) in this case $\lambda_{2} \sim m^{\alpha}$ with $1<\alpha<2$, whereas as explained above the small parameter of the theory is $m^{2}$. Expanding (4.16) in $Z \ll 1$, yields

$$
\left.\psi\right|_{\hat{d} \geq 5}=\bar{\psi}\left(1+\left(\frac{\rho_{0}}{\rho}\right)^{\hat{d}+4} \frac{\Gamma^{2}(1+2 / \hat{d})}{\Gamma(3 / 2+2 / \hat{d}) \Gamma(1 / 2+2 / \hat{d})} \tan (2 \pi / \hat{d})+\ldots\right) .
$$

Matching with the EFT result, we obtain

$$
\lambda_{2}=\frac{\pi^{\hat{d} / 2+1} \Gamma^{2}(1+2 / \hat{d})}{\Gamma(\hat{d} / 2+2) \Gamma(3 / 2+2 / \hat{d}) \Gamma(1 / 2+2 / \hat{d})} \tan (2 \pi / \hat{d}) \rho_{0}^{\hat{d}+4} .
$$

This result agrees with (4.17) upon substituting $l=2$ and taking into account (3.4).

The divergence at $l=2, d=7$. Let us now consider $\hat{d}=4$, namely $l=2$ in $7 \mathrm{~d} .{ }^{16} \mathrm{In}$ this case we should include the diagram in figure 6(b), since $\lambda_{2} \sim m^{2}$

$$
\text { Fig.6(b) }=\bar{\psi}\left(\frac{\rho_{0}}{\rho}\right)^{2 \hat{d}} \frac{\Gamma(2-\hat{d} / 2)}{\Gamma(3-\hat{d} / 2)} .
$$

This diagram diverges in $\hat{d}=4$. Thus in the language of quantum field theory, we have to renormalize the indefinite (bare) parameter $\lambda_{2}$ such that $\psi$ becomes finite. We now demonstrate this procedure.

Using (5.3) to expand figure $6(\mathrm{~b})$ in $\epsilon$, yields

$$
\text { Fig.6(b) }=-\frac{\bar{\psi}}{4}\left(\frac{\rho_{0}}{\rho}\right)^{8}\left(\frac{8}{\epsilon}+\frac{44}{5}-8 \gamma-8 \log \pi-16 \log \frac{\rho}{L}+\mathcal{O}(\epsilon)\right) .
$$

where $\gamma$ is the Euler constant. To cancel the pole in $\epsilon$, we redefine $\lambda_{2}$ as follows

$$
\lambda_{2}=L^{\epsilon} \rho_{0}^{8}\left(\lambda_{2}^{(0)}+\frac{\lambda_{2}^{(1)}}{\epsilon}+\frac{\lambda_{2}^{(2)}}{\epsilon^{2}}+\text { other possible poles in } \epsilon\right),
$$

where $\lambda_{2}^{(i)}, i>1$ are $\epsilon$-independent constants adjusted to eliminate all poles in $\epsilon$ which appear in $\psi$, while $\lambda_{2}^{(0)}$ depends on an arbitrary scale $L$ and should be matched to agree with the full solution in this case. This is the so called minimal subtraction (MS) scheme.

Pictorially, the above redefinition can be depicted as in figure 7 . Namely, one can think about it as decomposition of the bare parameter $\lambda_{2}$ into a finite piece proportional to $\lambda_{2}^{(0)}$ and an infinite part associated with $\lambda_{2}^{(1)}$. In the QFT literature the latter is called a counter term.

Substituting (6.10) into (6.5) and expanding in $\epsilon$, yields

Fig. $6(\mathrm{a})=\frac{\bar{\psi}}{\pi^{3}}\left(\frac{\rho_{0}}{\rho}\right)^{8}\left[\frac{6 \lambda_{2}^{(1)}}{\epsilon}+6 \lambda_{2}^{(0)}+\frac{11}{2} \lambda_{2}^{(1)}-3 \lambda_{2}^{(1)} \gamma-3 \lambda_{2}^{(1)} \log \pi-6 \lambda_{2}^{(1)} \log \frac{\rho}{L}+\mathcal{O}(\epsilon)\right]$.

\footnotetext{
${ }^{16}$ The more general case of $\hat{l}=1 / 2$ with arbitrary $d$ is quite similar.
} 

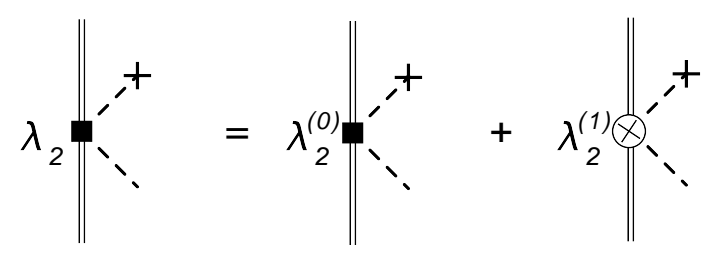

(a)

(b)

Figure 7. Pictorial representation of decomposition of (6.10). (a) represents the finite part, whereas (b) corresponds to an infinite counter term.

To cancel the pole in (6.9), we choose

$$
\lambda_{2}^{(1)}=\frac{\pi^{3}}{3}
$$

Hence,

$$
\begin{aligned}
\left.\psi_{E F T}\right|_{\hat{d}=4} & =\text { Fig.6(a) }+ \text { Fig.6(b) }+\ldots \\
& =\bar{\psi}\left[1+\left(\frac{\rho_{0}}{\rho}\right)^{8}\left(\frac{6}{\pi^{3}} \lambda_{2}^{(0)}-\frac{11}{30}+\gamma+\log \pi+2 \log \frac{\rho}{L}+\mathcal{O}(\epsilon)\right)+\ldots\right] .
\end{aligned}
$$

On the other hand, expanding the full solution (4.16) in the vicinity of $\hat{d}=4$, we obtain

$$
\left.\psi\right|_{\hat{d}=4}=\bar{\psi}\left[1+\left(\frac{\rho_{0}}{\rho}\right)^{8}\left(\frac{4 \log 2-1}{4}+2 \log \frac{\rho}{\rho_{0}}\right)+\ldots\right] .
$$

First thing to note is that EFT correctly reproduces the leading $\log \rho$ term. Finally, matching the results, yields

$$
\begin{aligned}
\lambda_{2}^{(0)}(L) & =\left.\lambda_{2}^{(0)}\right|_{L=\rho_{0}}+\frac{\pi^{3}}{3} \log \frac{L}{\rho_{0}}, \\
\left.\lambda_{2}^{(0)}\right|_{L=\rho_{0}} & =\frac{\pi^{3}}{6}\left(\log \frac{2}{\pi}+\frac{7}{60}-\gamma\right) .
\end{aligned}
$$

The corresponding classical RG flow is thus given by

$$
\beta_{\lambda_{2}}=L \frac{d \lambda_{2}^{(0)}}{d L}=\frac{\pi^{3}}{3}
$$

All the above results are inherent in our master formula (4.17) as is evident from (5.6).

Subleading corrections. When $\hat{d}=2,3$ there is no need to compute new diagrams. Indeed, in this case $\lambda_{2} \sim m^{\alpha}$ with $2<\alpha \leq 3$, therefore only diagrams in figures 6(a) and 
6(b) are relevant for our needs. Using (6.5) and (6.8), we obtain

$$
\begin{aligned}
& \left.\psi_{E F T}\right|_{\hat{d}=2}=\bar{\psi}\left[1+\left(\frac{\rho_{0}}{\rho}\right)^{4}+\frac{2}{\pi^{2}} \frac{\lambda_{2}}{\rho^{6}}+\ldots\right] \text {, } \\
& \left.\psi_{E F T}\right|_{\hat{d}=3}=\bar{\psi}\left[1+2\left(\frac{\rho_{0}}{\rho}\right)^{6}+\frac{15}{8 \pi^{2}} \frac{\lambda_{2}}{\rho^{7}}+\ldots\right] \text {. }
\end{aligned}
$$

Comparing with the asymptotic expansion of the full solution (4.16), yields

$$
\begin{aligned}
& \left.\lambda_{2}\right|_{\hat{d}=2}=0 \\
& \left.\lambda_{2}\right|_{\hat{d}=3}=-\frac{8 \pi^{2} \sqrt{3} \Gamma^{2}(5 / 3)}{15 \Gamma(7 / 6) \Gamma(13 / 6)} \rho_{0}^{7} .
\end{aligned}
$$

These results agree with (4.17).

The last case to consider is $\hat{d}=1$. In this case, the full solution degenerates into a polynomial of fourth order in $Z$

$$
\left.\psi\right|_{\hat{d}=1}=\bar{\psi}\left[1+\frac{2}{3}\left(\frac{\rho_{0}}{\rho}\right)^{2}+\left(\frac{\rho_{0}}{\rho}\right)^{4}\right] .
$$

In particular, there is no need in any computation to fix $\lambda_{2}$. Indeed, since as argued above, in isotropic coordinates the small parameter is $\mathrm{m}^{2}$, whereas according to (2.13) $\lambda_{2} \sim m^{5}$ in $\hat{d}=1$, we conclude that there are no diagrams with mass insertions which scale in the same way as figure 6(a). Furthermore, the full solution does not contain odd powers of $m \sim \rho_{0}$. As a result, we conclude that $\lambda_{2}$ must be zero to match the full solution in this case. Of course, this result fits our general formula (4.17).

It is instructive to show how the EFT reproduces the full solution (6.21). This requires computation of one additional diagram apart from what we have computed so far, i.e. figure $6(\mathrm{c})$

$$
\operatorname{Fig} .6(\mathrm{c})=-\frac{3(\hat{d}-2)}{(\hat{d}-4)(3 \hat{d}-4)} \bar{\psi}\left(\frac{\rho_{0}}{\rho}\right)^{4 \hat{d}} .
$$

For $\hat{d}=1$ this diagram reproduces the last term in (6.21). Note also that as expected it vanishes in $\hat{d}=2$. Indeed, in this case the full solution (6.21) truncates at second order in $Z$, therefore the overall contribution at order $Z^{4}$ must vanish. As a last test of this result we checked that it produces correct contribution in $\hat{d}=3$.

\section{$7 \quad$ Summary of results}

Results. Our main result is the determination of the induced gravito-static polarization constants, or gravito-electric Love numbers, for any space-time dimension $d$, given by (4.23) together with the text below it and the normalization factor (3.5).

We also computed the Love numbers for a free scalar field given essentially by (4.17). In $d>4$ these describe also the tensor mode perturbations of the spatial metric. 
Tests. The results passed two tests

- The $4 \mathrm{~d}$ results are consistent with $[8,9]$. Their vanishing is dictated by the $\tan \pi \hat{l}$ factor.

- The residue of the pole at $\hat{l}=1 / 2$ was compared with the EFT thereby confirming the overall normalization factor.

\section{Main implications.}

- We settled a disagreement in the literature whether the calculation described in [8] and [9] implies a vanishing Love number or not. We find that in hindsight it indeed implies so and we supply the missing argument. This means that even though the horizons of $4 \mathrm{~d}$ black holes do deform they are nevertheless "infinitely rigid" in this sense.

- We answered negatively (at least for $d>4$ ) a conjecture about the absence of nonminimal world-line couplings in the $\mathrm{BH}$ effective action.

- Our results for higher dimensions $d>4$ are unquestionably novel, as well as those for the free scalar field.

- We observed that the Love numbers are negative for certain ranges of parameters and interpreted that as indication for a novel non-spherical instability.

See more in the discussion subsection 4.2.

On the way we touched upon the following points of interest

- We defined the static sector of the black hole effective action (2.2).

- We defined $\hat{l}$ (3.7) and noticed that most of the analysis depends on $l, d$ only through it.

- We found quite a simple action (4.21) which encodes the full Zerilli potential.

- We noted that there is an interesting limit to the theory as $\hat{l} \rightarrow \infty$ where only the $\tan \pi \hat{l}$ term survives in (4.23).

- We provided a nice example of a classical RG flow, analyzed in sections 5,6.

\section{Acknowledgments}

A significant part of BK's work was carried in the pleasant environment of the workshop "Gravity - New perspectives from strings and higher dimensions" (July 2011, Benasque). BK appreciates the hospitality of the meeting "the 6th regional meeting on string theory" (June 2011, Milos) and of G. Schäfer at Jena University (Aug 2011). 
This research was partly supported by the Israel Science Foundation grants no 607/05 and $812 / 11$, by the German Israel Cooperation Project grant DIP H.52, and by the Einstein Center at the Hebrew University, though for most of the relevant time BK was not supported by research grants. Research at Perimeter Institute is supported by the Government of Canada through Industry Canada and by the Province of Ontario through the Ministry of Research \& Innovation.

\section{A Matching normalization constant}

In this appendix we evaluate the normalization constant $N(3.4)$. This is done by solving the EFT equation of motion in the presence of the $\lambda$ term (2.9) (obviously in flat spacetime) and reading off the $\hat{\lambda}$ coefficient defined in (3.2).

The relevant part in the EFT action reads

$$
S \supset-\frac{1}{2} \int \partial_{i} \psi \partial_{i} \psi+\frac{1}{2} \lambda_{l}\left|\partial^{I_{l}} \psi\right|^{2}
$$

where $\left|\partial^{I_{l}} \psi\right|^{2}$ is defined in (2.9). The following analysis does not change by a multiplication of the action by an overall constant. The equation of motion is

$$
0=\triangle \psi+\left.\frac{\lambda_{l}}{l !} \sum_{I_{l}} \partial_{I_{l}} \psi\right|_{0} \partial_{I_{l}} \delta(-x)
$$

Following (3.2) we write the solution as

$$
\psi=\frac{1}{l !} \sum \psi_{I_{l}} x^{I_{l}}\left(1+\frac{\hat{\lambda}}{r^{2 l+\hat{d}}}\right)
$$

Substituting back into (A.2) we find that

$$
N=(-)^{l}\left(c_{0} c_{1}\right)^{-1}
$$

where

- $c_{0}$ is defined to be such that

$$
\triangle\left(-\frac{c_{0}}{r^{\hat{d}}}\right)=\delta(x)
$$

namely, $G_{0}=-c_{0} / r^{\hat{d}}$ is a Green function satisfying $\triangle G_{0}=\delta(x)$. We have

$$
c_{0}=\frac{1}{\hat{d} \Omega_{\hat{d}+1}}=\frac{\Gamma\left(\frac{\hat{d}}{2}\right)}{4 \pi^{\frac{\hat{d}}{2}+1}} .
$$

where $\Omega_{\hat{d}+1}:=\operatorname{Vol}\left(\mathbf{S}^{\hat{d}+1}\right)$ 
- $c_{1}$ is defined such that

$$
\partial_{I} \frac{1}{r^{\hat{d}}}=c_{1} \frac{x^{I}}{r^{2 l+\hat{d}}}
$$

where $I=\left(i_{1}, \ldots, i_{l}\right)$ and all the indices $i_{1}, \ldots, i_{l}$ are distinct. We find

$$
c_{1}=(-2)^{l}\left(\frac{\hat{d}}{2}\right)_{l}=(-2)^{l} \frac{\Gamma\left(\frac{\hat{d}}{2}+l\right)}{\Gamma\left(\frac{\hat{d}}{2}\right)}
$$

Substituting back into (A.4) we obtain the requested quantity

$$
N=\frac{\hat{d} \Omega_{\hat{d}+1}}{2^{l}\left(\frac{\hat{d}}{2}\right)_{l}}=\frac{\pi^{\frac{\hat{d}}{2}+1}}{2^{l-2} \Gamma\left(\frac{\hat{d}}{2}+l\right)}
$$

\section{B Useful formulae}

We collect some useful facts for the microscopic calculation, starting with the hypergeometric function, see for example [21].

The hypergeometric equation for $u=u(y)$ is

$$
y(1-y) u^{\prime \prime}+[c-(a+b+1) y] u^{\prime}-a b u=0
$$

The characteristic exponents are encoded in the Riemann P-symbol

$$
u(y)=P\left(\begin{array}{ccc}
0 & 1 & \infty \\
0 & 0 & a ; y \\
1-c & c-a-b & b
\end{array}\right)
$$

The hypergeometric series is defined by

$$
\begin{aligned}
F(a, b, c ; x) & :=\sum_{k=0}^{\infty} \frac{(a)_{k}(b)_{k}}{(c)_{k} k !} x^{k} \\
(a)_{k} & :=\frac{\Gamma(a+k)}{\Gamma(a)} \equiv a \cdot(a+1) \ldots(a+k-1)
\end{aligned}
$$

Pfaff's identity is

$$
F(a, b, c ; X)=(1-X)^{-b} F\left(c-a, b, c ; \frac{X}{X-1}\right)
$$

Expansion around $X=0$ is given by [21](8.3.6)

$$
\begin{aligned}
F(a, b, c ; 1-X) & =\frac{\Gamma(c) \Gamma(c-a-b)}{\Gamma(c-a) \Gamma(c-b)} F(a, b, a+b+1-c ; X)+ \\
& +\frac{\Gamma(c) \Gamma(a+b-c)}{\Gamma(a) \Gamma(b)} X^{c-a-b} F(c-a, c-b, 1+c-a-b ; X)
\end{aligned}
$$


The Legendre polynomial is related to the hypergeometric function through

$$
P_{l}(x)=F\left(-l, l+1,1 ; \frac{1-x}{2}\right)
$$

Finally we record some useful Gamma function identities

$$
\begin{aligned}
\Gamma(x) \Gamma(1-x) & =\frac{\pi}{\sin \pi x} \\
\Gamma(x) \Gamma\left(x+\frac{1}{2}\right) & =2^{1-2 x} \sqrt{\pi} \Gamma(2 x)
\end{aligned}
$$

\section{References}

[1] The Oxford English dictionary www.oed.com, retrieved on Sep 12, 2011.

[2] A.E.H. Love, "Some problems of geodynamics," Cambridge University Press (1911) and re-published by Dover, New York (1967).

See also http://en.wikipedia.org/wiki/Augustus_Edward_Hough_Love

[3] Y. -Z. Chu, W. D. Goldberger, I. Z. Rothstein, "Asymptotics of d-dimensional Kaluza-Klein black holes: Beyond the Newtonian approximation," JHEP 0603, 013 (2006).

[hep-th/0602016].

[4] B. Kol and M. Smolkin, "Classical Effective Field Theory and Caged Black Holes," Phys. Rev. D 77, 064033 (2008) [arXiv:0712.2822 [hep-th]].

[5] J. B. Gilmore, A. Ross, M. Smolkin, "Caged black hole thermodynamics: Charge, the extremal limit, and finite size effects," JHEP 0909, 104 (2009). [arXiv:0908.3490 [hep-th]].

[6] K. Schwarzschild, "On The Gravitational Field Of A Mass Point According To Einstein's Theory," Sitzungsber. Preuss. Akad. Wiss. Berlin (Math. Phys. ) 1916, 189 (1916) [arXiv:physics/9905030].

[7] E. E. Flanagan, T. Hinderer, "Constraining neutron star tidal Love numbers with gravitational wave detectors," Phys. Rev. D77, 021502 (2008). [arXiv:0709.1915 [astro-ph]].

[8] T. Damour, A. Nagar, "Relativistic tidal properties of neutron stars," Phys. Rev. D80, 084035 (2009). [arXiv:0906.0096 [gr-qc]].

[9] T. Binnington, E. Poisson, "Relativistic theory of tidal Love numbers," Phys. Rev. D80, 084018 (2009). [arXiv:0906.1366 [gr-qc]].

[10] T. Damour, O. M. Lecian, "On the gravitational polarizability of black holes," Phys. Rev. D80, 044017 (2009). [arXiv:0906.3003 [gr-qc]].

[11] W. D. Goldberger and I. Z. Rothstein, "An effective field theory of gravity for extended objects," Phys. Rev. D 73, 104029 (2006) [arXiv:hep-th/0409156].

[12] W. D. Goldberger, "Les Houches lectures on effective field theories and gravitational radiation," arXiv:hep-ph/0701129.

[13] T. Damour and G. Esposito-Farese, "Testing gravity to second postNewtonian order: A Field theory approach," Phys. Rev. D 53, 5541 (1996) [arXiv:gr-qc/9506063].

[14] V. Asnin, D. Gorbonos, S. Hadar, B. Kol, M. Levi, U. Miyamoto, "High and Low Dimensions in The Black Hole Negative Mode," Class. Quant. Grav. 24, 5527-5540 (2007). [arXiv:0706.1555 [hep-th]]. 
[15] B. Kol, E. Sorkin, "On black-brane instability in an arbitrary dimension," Class. Quant. Grav. 21, 4793-4804 (2004). [gr-qc/0407058].

[16] W. D. Goldberger, I. Z. Rothstein, "Dissipative effects in the worldline approach to black hole dynamics," Phys. Rev. D73, 104030 (2006). [hep-th/0511133].

[17] B. Kol, "The Delocalized Effective Degrees of Freedom of a Black Hole at Low Frequencies," Gen. Rel. Grav. 40, 2061-2068 (2008). [arXiv:0804.0187 [hep-th]].

[18] B. Kol and M. Smolkin, "Non-Relativistic Gravitation: From Newton to Einstein and Back," Class. Quant. Grav. 25, 145011 (2008) [arXiv:0712.4116 [hep-th]].

[19] H. Kodama, A. Ishibashi, "A Master equation for gravitational perturbations of maximally symmetric black holes in higher dimensions," Prog. Theor. Phys. 110, 701-722 (2003). [hep-th/0305147].

[20] A. Ishibashi, H. Kodama, "Stability of higher dimensional Schwarzschild black holes," Prog. Theor. Phys. 110, 901-919 (2003). [hep-th/0305185].

[21] R. Beals and R. Wong, Special functions, Cambridge University Press (2010).

[22] F. J. Zerilli, "Effective Potential For Even Parity Regge-Wheeler Gravitational Perturbation Equations," Phys. Rev. Lett. 24, 737 (1970).

[23] V. Asnin, B. Kol, "Dynamical versus auxiliary fields in gravitational waves around a black hole," Class. Quant. Grav. 24, 4915-4935 (2007). [hep-th/0703283].

[24] B. Kol, "Perturbations around backgrounds with one non-homogeneous dimension," [hep-th/0609001].

[25] D. Gorbonos and B. Kol, "A dialogue of multipoles: Matched asymptotic expansion for caged black holes," JHEP 0406, 053 (2004) [arXiv:hep-th/0406002].

[26] I. Vega, E. Poisson, R. Massey, "Intrinsic and extrinsic geometries of a tidally deformed black hole," Class. Quant. Grav. 28, 175006 (2011). [arXiv:1106.0510 [gr-qc]].

[27] R. Gregory and R. Laflamme, "Black strings and p-branes are unstable," Phys. Rev. Lett. 70, 2837 (1993) [arXiv:hep-th/9301052].

[28] B. Kol and M. Smolkin, "Dressing the Post-Newtonian two-body problem and Classical Effective Field Theory," Phys. Rev. D 80, 124044 (2009) [arXiv:0910.5222 [hep-th]].

[29] W. D. Goldberger, A. Ross, "Gravitational radiative corrections from effective field theory," Phys. Rev. D81, 124015 (2010). [arXiv:0912.4254 [gr-qc]]. 